\title{
A Modular Robot System Design and Control Motion Modes for Locomotion and Manipulation Tasks
}

\author{
José Baca and Manuel Ferre and Rafael Aracil and Alexandre Campos
}

\begin{abstract}
This paper describes a modular robot system design SMART, based on three types of modules for urban search tasks. The system attempts to give a quick solution to natural and man-made disaster emergencies. It allows for rapid and cost-effective design and fabrication. The approach is based on the use of an inventory of three types of modules i.e., power and control module, joint module, and specialized module. They are interchangeable in different ways to form different robot configurations for a variety of tasks. Forward and inverse kinematics from assembled robot configurations are analyzed. Description of control motion modes for humanmodular robot system interaction is presented.
\end{abstract}

\section{INTRODUCTION}

Weather-related disasters are impacting mankind with relentless frequency and intensity and have taken a heavy toll in recent years. Earthquakes, tsunamis, floods, and hurricanes are devastating cities around the world. Similarly, man-made disasters caused by fires, explosions, structural imperfections and wars constitute a clear and ever present danger for mankind. On January 12th, 2010 an earthquake of a magnitude of 7.2 in the Richter scale hit Haiti, by the morning of January 14th reporters were talking of tens, if not hundreds, of thousands of lives lost at schools, hospitals, houses, offices, shops, and headquarters of the United Nations. All the infrastructure collapsed in those $\mathbf{4 5}$ murderous seconds. On February of 2010 , earthquakes of great magnitude hit Japan and Chile, and lately floods, snowstorms, etc., around the world confirm a trend towards an increase in the number of natural catastrophes and man-made disasters,

Nowadays, robots are commonly used for technical search tasks in Urban Search and Rescue (USAR) [1]. The motivation for rescue robots is varied, e.g., miniature robots that can go into places that living things cannot due to size, extreme heat, toxicity of the environment, rescue safety, effectiveness, etc. A robot can be deployed in minutes, there are not enough trained individuals to perform the multitude of tasks during a rescue: search, extract, examine, inspect, and medically treat, etc. The robot platforms for USAR vary widely in terms of size, type of mobility (wheels, tracks, or combination), and ruggedness. In [2] and [3], the idea of marsupial and shape-shifting robots for USAR is explored. In [5] and [12], biologically inspired snake robot platforms for USAR are explored. Fire rescue and outdoor robot developments are investigated in [4], [6]-[11]. Software development for USAR has involved creating software for robot control, multirobot

This work was supported by the Spanish Ministerio de Educacin, under Grant DPI 2003-00759 and DPI-2006-06493 and CONACYT from Mexico.

J. Baca is with the Group of Intelligent Machines. Universidad Politécnica de Madrid, Madrid, Spain, jbaca etsii.upm.es collaboration, multisensory control, and aiding humans using robot equipment. In [2], the idea of automated behaviors for shape-shifting robots is presented. Collaborative USAR robots are explored in [13]. A multiple sensor control system on a USAR robot is investigated in [14]. Software developed to aid the human operator through an intelligent expert system and mixed-initiative system is described in [15]-[17]. Despite the great advantages that show this type of robotic system, they have disadvantages, e.g., the long development time, high initial costs and most of them are designed for a specific mission, high power requirement/low run time (if not tethered). These disadvantages stop the rapid construction of robots that try to help in the rescue operations. An increment in the number of natural disasters is a fact, therefore, human rescue teams urgently require a wide variety of robots that can help in different situations. A quick and effective response to a developing urgent situation is the number one way to save lives and property. Disasters can strike like a flash, fast and without warning.

On the other hand, modular robots systems are usually an inventory of physical robotic modules that may be assembled in different configurations to perform different tasks. This type of system is composed of multiple units of a relatively small repertoire, with docking interfaces that allow transfer of mechanical forces and moments, electrical power, and communication throughout the robot. Most of the work in this area involves identical modules with interconnection mechanisms that allow either manual or automatic reconfiguration. It is possible to find modular robot designs from 1 degree of freedom (DOF) [18], 2 DOF [19], [20], 3 DOF [21], and designs with passive joints [22]. Some systems employ a cube-type arrangement, with modules which are able to connect in various ways to form matrices or lattices for specific functions. Other types of work involve the simulation of an inventory of different modules that may be reconfigured to perform different tasks [23].

In section II a modular robot system design based on three interchangeable types of modules for USAR robots is proposed. The system attempts to give a quick solution to natural and man-made disaster emergencies. It allows for rapid and cost-effective design and fabrication. The approach is based on the use of an inventory of three types of modules i.e., power and control module, joint module, and specialized module. Possible robot configurations that may be assembled with the modules are presented in section III with some kinematic analysis in section IV. The system also uses software modules that are downloaded into the module to behave according to the robot configuration. Like in human- 
robot interfaces proposed by teleoperated USAR robots [24], we describe in section V modular motion modes to facilitate human-robot interaction during teleoperated tasks.

\section{ROBOT MODULES DESCRIPTION}

The system architecture is divided into modules, M-Robots and colonies. Modules are base system components and are classified in three types of modules, i.e., power/control module, joint module and specialized module as shown in Fig. 1.

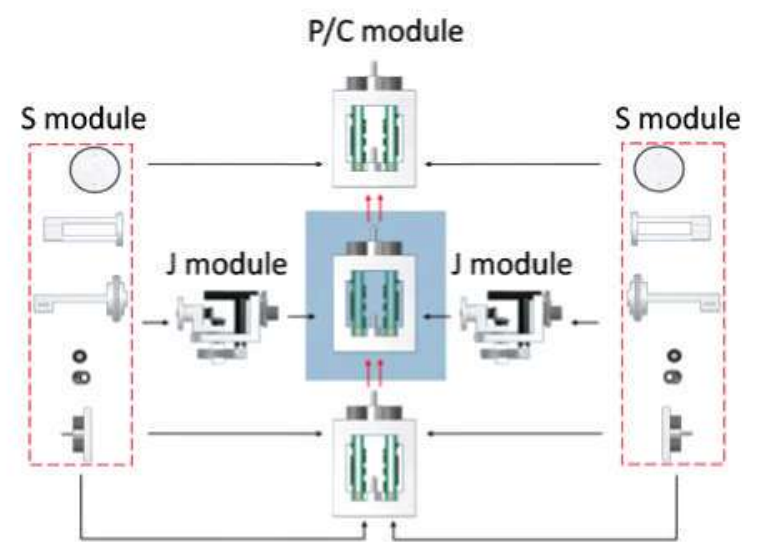

Fig. 1. Three types of modules may be combined to form different robot configurations

The designed modules for the system aim to balance complexity and functionality. The purpose is to build robots that offer movement flexibility, allowing a variety of locomotion modes and reconfiguration capabilities. According to the control system, it uses a centralized architecture because high speed communication exists within the modules aiming for synchronization among them.

\section{A. Power/Control module}

As its name suggests, this module contains the electronic boards, the system power source, the communication peripherals and mechanisms to physically connect other types of modules. The module introduced in this paper is a $80 \mathrm{~mm} x$ $150 \mathrm{~mm} \times 55 \mathrm{~mm}$ cuboid (Fig. 2) which contains the system power source (a rechargeable lithium battery), two electronic boards and four connectors (two permanent and two nonpermanent connectors).

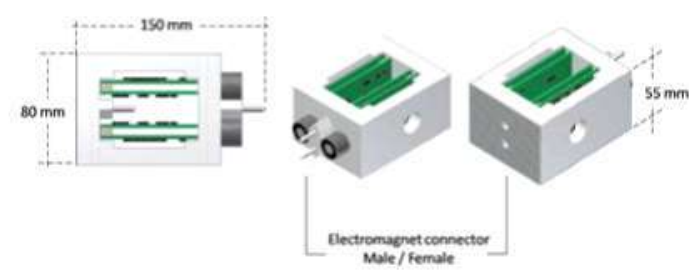

Fig. 2. Power/Control (P/C) module

Non-permanent connectors join $P / C$ modules and are used for reconfiguration. It consists of male and female connectors. The male connector has two electromagnetic devices controlled by the electronic board and two contact points that transfer CAN signals between $P / C$ modules. The female connector is a metallic plate with two contact points that transfer CAN signals between $P / C$ modules, see Fig. 3. Permanent connectors join a $P / C$ module with joint or specialized modules manually (screwed), see Fig. 3.d. The $P / C$ module may be docked up to two $P / C$ modules, using the non-permanent connector and up to two modules (joint or specialized modules) using the permanent connector, as shown in Fig.1.

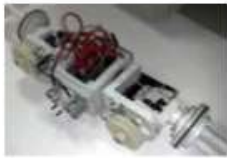

(a)

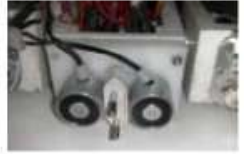

(b)

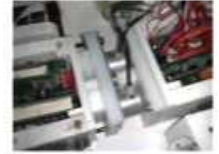

(c)

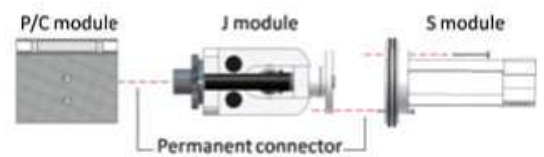

(d)

Fig. 3. (a) Detail and distribution of non-permanent connectors: electromagnet and plate in a $P / C$ module. (b) Male connector based on two electromagnets. (c) Connector detail of two joined $P / C$ modules. (d) Permanent connector.

\section{B. Joint module}

The joint $(J)$ module represents any type of active or passive actuator such as linear, rotary, pneumatic hydraulic, spherical, etc. In this case, an actuator with 3 rotational degrees of freedom whose axes intersect at one point is studied. Figure 4 shows the actuator. The design of the $J$ module allows $S$ or $P / C$ modules to be attached/detached, according to the task, through the permanent connector at its ends. Depending on the task, different configurations may be used. In order to have this module working, it must contain a $P / C$ module at one of its ends. This is a robot assembly rule.

\section{Specialized module}

The specialized $(S)$ module may be described as the endeffector of the robot. It may be a limb type (e.g., leg $(L)$, wheel $(W)$ or hybrid limb $(H)$ ), a sensor device, a tool, an accessory (e.g., platform) or a connector. This type of module can be attached to a $P / C$ module, $J$ module or
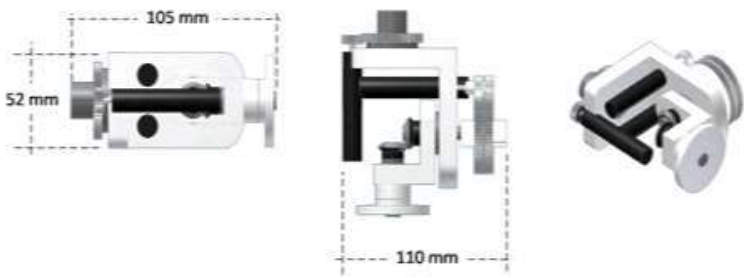

Fig. 4. Joint module, spherical actuator 


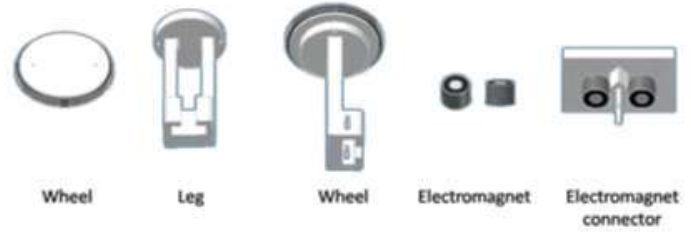

Fig. 5. Specialized modules

another $S$ module. The $S$ module provides more flexibility and diversity to locomotion and manipulation tasks, a few examples of $\mathrm{S}$ modules are shown in Fig. 5.

\section{MODULAR ROBOT CONFIGURATIONS}

The advantage of having interchangeable modules is the ability to build different robot configurations. Depending on the mission, the operator decides what type of robot can provide the best performance within the mission. The module inventory provides a variety of possibilities when assembling the robot.

\section{A. M-Robot}

An $M$-Robot is an autonomous entity made up of at least one module of each type $(P / C, J$ and $S)$, see Fig. 6. A general M-Robot configuration contains a $P / C$ module, $J$ modules and $S$ modules. It is named as $T n \mathrm{M}$-Robot, where $n=2,4,6, \cdots$ represents the number of $J$ modules used in the robot configuration and $T=\mathrm{L}, \mathrm{W}, \mathrm{H}$ etc., represents the $S$ module type: leg, wheel or hybrid, respectively. Basic TnMRobot configurations are made up of five modules named as $T 2 M$-Robot, i.e., one $P / C$ module, two $J$ modules and two $S$ modules. For instance the configurations L2M-Robot, W2M-Robot and H2M-Robot are shown in Fig. 7.

\section{B. Complex M-Robot configurations}

If two or more T2M-Robots are docked together, through non-permanent connectors, they form a new robot structure named T4M-Robot, T6M-Robot, etc. These robot configurations provide new capabilities for objects handling and motion. For instance, if two $L 2 M$-Robots are connected to each other, a $L 4 M$-Robot is assembled.

If three W2M-Robots are connected together, a W6M-Robot is obtained. In general, connecting basic T2M-Robots of different $S$ modules to perform special task could be done.

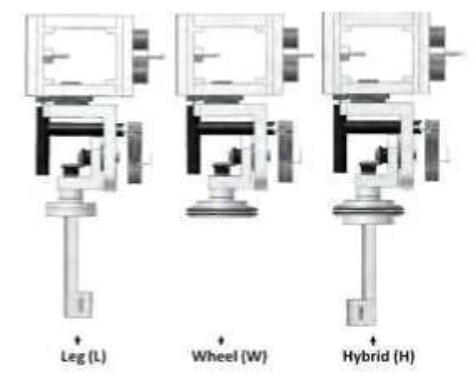

Fig. 6. Basic M-Robot with different $S$ modules

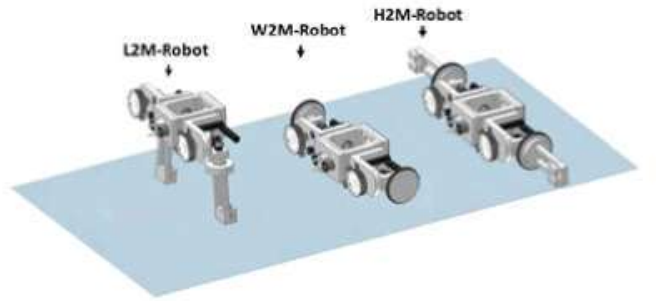

Fig. 7. M-Robot configurations: L2M-Robot,W2M-Robot and H2M-Robot

Furthermore, a hybrid complex M-Robot complies with all these requirements due to its changing flexibility between leg and wheel, see Fig. 8.

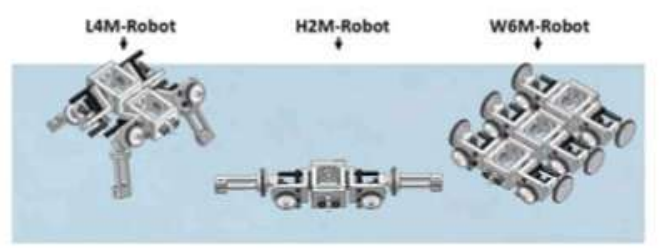

Fig. 8. Complex M-Robot configurations: L4M-Robot,H2M-Robot and W6M-Robot

Several M-Robots may be assembled through different module combinations, for instance see Fig. 9. Complex MRobots hold higher capability of movement, communication and manipulation. Let a colony be defined as various MRobots cooperating together to fulfill a task.

Therefore, according to the classification of modular robots, the modular system may be classified as heterogeneous since it contains different types of modules. Regarding the connectors'position, the system may be classified as a chain (serial) type modular robot due to the electromagnetic connectors on $P / C$ modules. However, $J$ modules and $S$ modules may be assembled in parallel as shown in Fig. 9.

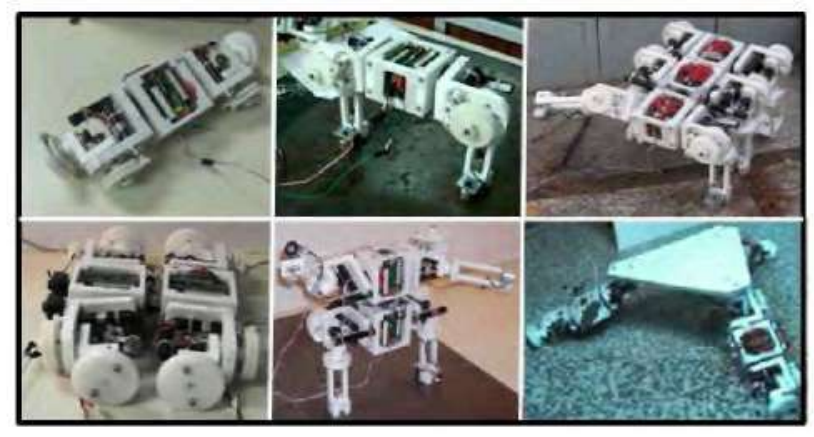

Fig. 9. By combining modules in different configurations, several M-Robots may be obtained

\section{KINEMATICS DESIGN}

The assembly of a $P / C$ module with a $J$ module may create a simple robot. The $J$ module under discussion is the spherical actuator, which has been docked with an $S$ 
module i.e., leg-wheel element. According to the established definitions, this robot configuration is called HM-Robot. The robot is formed by three rotational degrees of freedom whose axes intersect at one point. Figure 10 shows the robot configuration, the schematic view and Denavit-Hartenberg parameters.

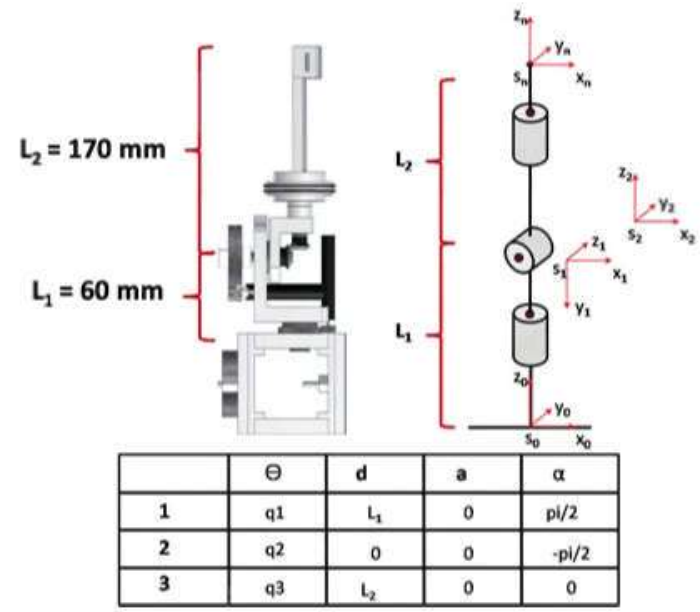

Fig. 10. HM-Robot scheme and its D-H parameters

The homogeneous transformation matrix (HTM) for the HM-Robot is shown in Eqn. 1. It gives the forward kinematics (FK) of the end-effector with respect to the frame at the union with the $P / C$ module body. In this paper the analysis of FK is used to propose modular motion modes that facilitate teleoperated tasks, this topic will be explained later on. Note that $s$ and $c$ are used to abbreviate sine and cosine, respectively.

$$
{ }_{3}^{0} T=\left[\begin{array}{cccc}
c 1 c 2 c 3-s 1 s 3 & -c 3 s 1-c 1 c 2 s 3 & -c 1 s 2 & -l 2 c 1 s 2 \\
c 1 s 3+c 2 c 3 s 1 & c 1 c 3-c 2 s 1 s 3 & -s 1 s 2 & -l 2 s 1 s 2 \\
c 3 s 2 & -s 2 s 3 & c 2 & l 1+l 2 c 2 \\
0 & 0 & 0 & 1
\end{array}\right]
$$

The inverse kinematics (IK) problem for one spherical actuator is extracted from the given HTM in Eqn. 1.

$$
\begin{aligned}
& \theta_{1}=\arctan \frac{p x}{p y} \\
& \theta_{2}=-\arctan \left(\frac{\cos \theta_{1} p x+\sin \theta_{1} p y}{p z-l_{1}}\right) \\
& \theta_{3}=-\arcsin \left(\sin \theta_{1} n x-\cos \theta_{1} n y\right)
\end{aligned}
$$

The workspace of the HM-Robot is illustrated in Fig. 11. As observed, the working area is a spherical surface. This characteristic will be translated into an advantage when carrying out displacement and manipulation tasks.

Adding certain modules to the HM-Robot, is possible to assemble a quadruped robot, as shown in Fig. 12. Two $P / C$ modules, four $J$ modules and four $S$ modules are sufficient modules to increase displacement capabilities. For instance, wheeled locomotion or legged locomotion may be performed using the same $S$ module. Kinematic analysis of this robot configuration is not difficult, due to identical $J$ modules and
3D Spherical actuator workspace

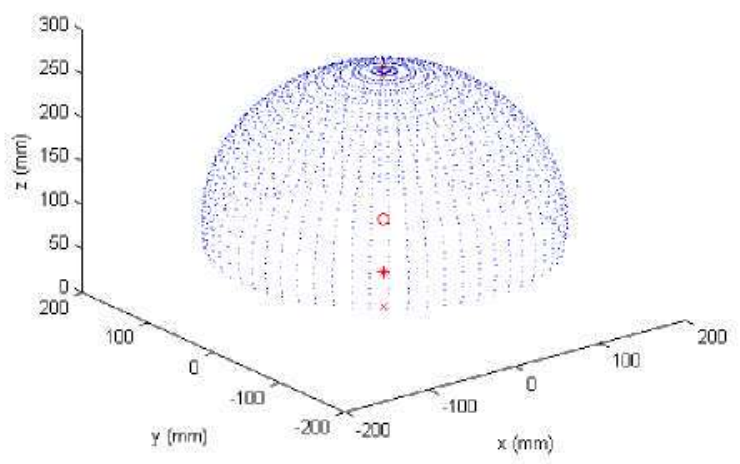

Fig. 11. Spherical actuator workspace

robot symmetry (the right-side legs are symmetric to the leftside legs). Applying the appropriate rotations and translations to eqn. 1 , it is possible to find the workspace of each of the ends of the new robot configuration as shown in Fig. 13. The symmetry of the robot allows us to use the same IK equations but angle signs must be considered for opposite side legs.

\section{HUMAN-MODULAR ROBOT INTERACTION}

For intuitive control of multiple robots by a single operator and for reproducing the intentions of the operator into the system, is necessary to provide the operator with tools that facilitate interaction between them. The integration of these features into interfaces has been continuously developed and tested. In contrast, modular robot systems have considerable capabilities which have to be fully exploited. Multiple robots'graphical user interfaces (GUI) mostly worry about increasing the understanding of the environment, but an interface for modular robot systems require the understanding of not only the surroundings, but also of its own reconfigurable mechanism during the mission.

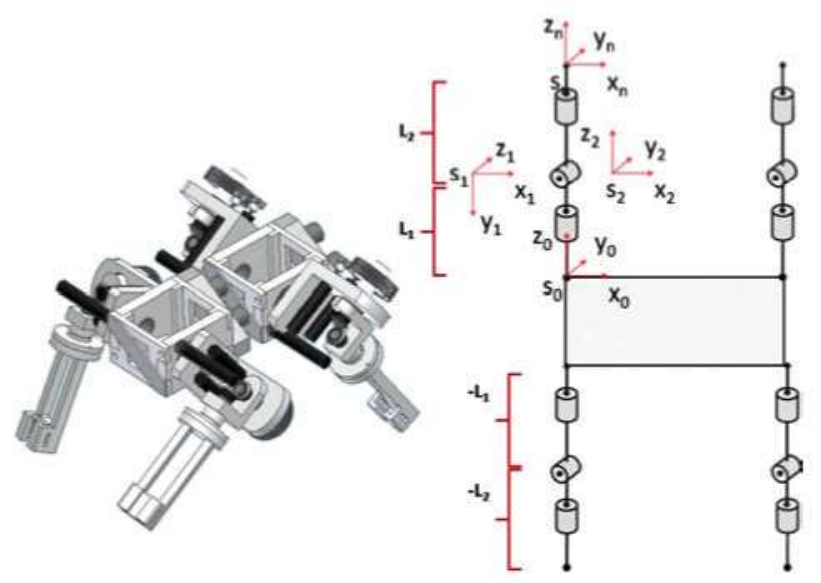

Fig. 12. H4M-Robot and its schematic top view 


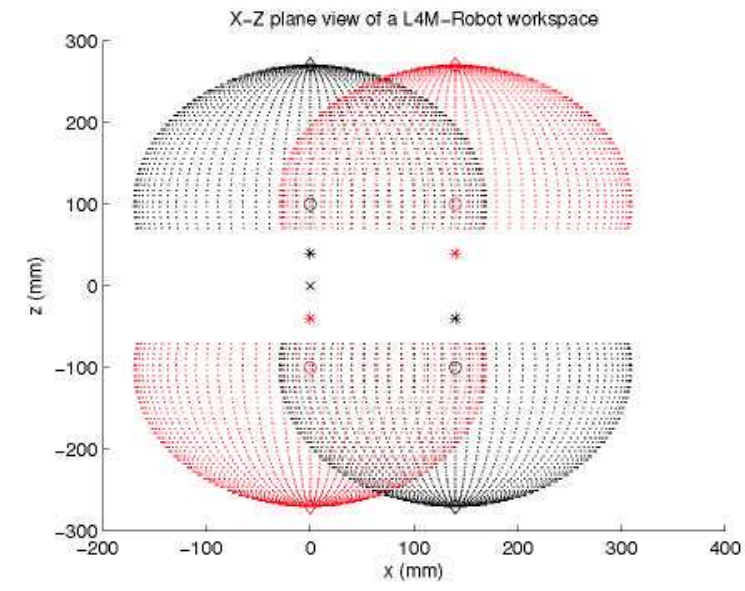

Fig. 13. H4M-Robot workspace allows wheeled and legged locomotion

Guidelines have been developed for the effective design of interfaces for human-robot interaction in search and rescue robots applications [25]. Modular robot systems may be controlled with a multiple robots GUI due to similar capabilities that they share. However, in the case of a modular robot configuration built of several modules, the control motion mode should or should not be that of a single robot. The great advantage of a modular robot system, is the ability to build different robot configurations within a wide range. It is important to note that certain configurations are effective when performing various tasks. For example, if the target of the mission is to reach a point $\mathrm{B}$ from a point $\mathrm{A}$, for instance, a mobile robot configuration would fit perfect for fast and efficient (energy consumption) displacement over regular and semi-regular surfaces. At some point between point $\mathrm{A}$ and point $\mathrm{B}$ an obstacle may interrupt the mobile robot and it is necessary to change its robot configuration from a mobile robot configuration to a four legged robot as shown in Fig. 14.1. Now, the robot can continue advancing and avoiding the obstacles step by step. After the obstacles have been passed, it is best to go back to the previous robot configuration to continue and reach the target, as shown in Fig. 14.2. This example clearly shows the need to provide the operator with certain modular robot motion modes to facilitate interaction within the robots.

\section{A. Modular Robot Motion Modes}

To teleoperate a specialized robot, it is normally required to have the robot kinematic model linked by a computer to a joystick or control pad. By this way, it moves the robot in different ways. Modular robot systems can be configured to have the functionality similar to that of a specialized robot. The complexity to teleoperate this type of system begins with the decision making of how to link each robot configuration to the control pad. For an intuitive control of a robot, behavior of single robot has to be achieved. In the case of a robot configuration built of several modules, it is imperative to define a way to control it. At some point in a mission, complicated circumstances e.g., an obstacle in the path, a narrow path, irregular surfaces, etc., may cause mission failure. Unlike specialized robots, modular robots must be capable of being reconfigured according to circumstances. Hence, it is essential to control the robot with a behavior related to its configuration. Typical circumstances are described bellow.

- The operator may be teleoperating for instance, a modular robot built of $2 \mathrm{P} / \mathrm{C}$ modules, $2 \mathrm{~J}$ modules and 2 $S$ modules, like the one shown in Fig. 15.1. The initial motion mode of this configuration is that of a legged robot. This motion mode is named Legged Mode and allows the robot to advance in certain directions moving its legs in a specific way. Biped, quadruped or hexapod robot are examples of robot configurations that require this type of modality.

- During the mission, an obstacle appears in front of the robot and the robot must pass over it. The operator requires the possibility to control a specific $J$ module with a behavior of that of a single module, as shown in Fig. 15.2. This motion mode is named Single Mode and allows control of the actuator in the chosen module.

- Now, imagine having a robot structure built of $1 \mathrm{P} / \mathrm{C}$ module, $2 J$ modules and $2 S$ modules and the task requires to grip an object, as shown in Fig. 15.3. In order to perform this typical manipulation task, an equal but opposite movement from both $J$ modules is required. To accomplish the task, Mirror Mode is suggested. If this behavior is applied for both modules, the operator only needs to control one module and the second module will reproduce the movement but in opposite direction, as shown in Fig. 15.4.

- Another case may be a mobile robot configuration. The operator requires a behavior of a mobile robot, i.e., if the operator presses forward, backward, left, or right in the control pad, the robot goes in that direction respectively rotating its wheels. This motion mode is named Mobile Mode and for instance, it may work for 2-wheel, 4wheel, or 6-wheel robot configurations, as shown in Fig. 15.5 and 15.6.

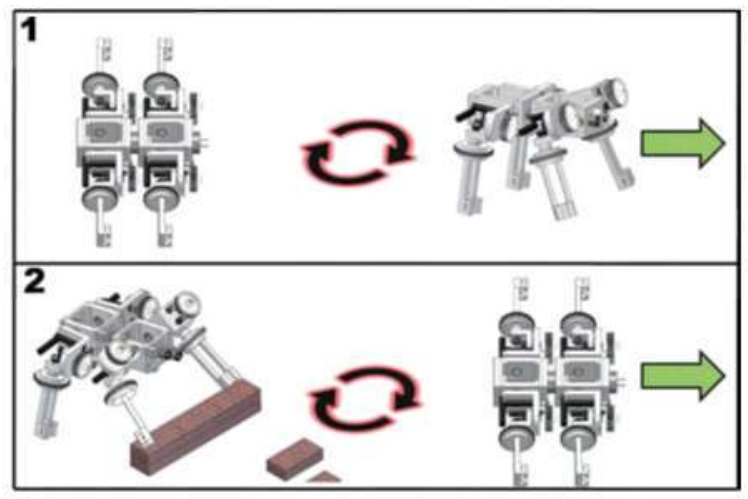

Fig. 14. During teleoperation tasks, useful configurations are often used. 


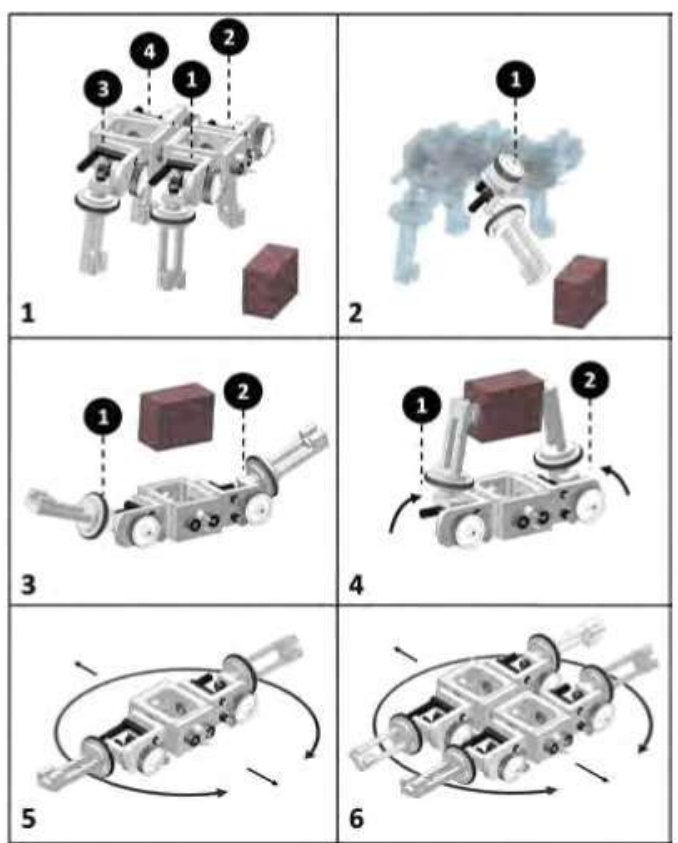

Fig. 15. Modular robots are capable of being reconfigured according to circumstances, thus, it is essential to control the robot with a behavior related to its configuration. Legged, single, mirror, and mobile modes are presented.

\section{CONCLUSION}

A modular robot system design based on three interchangeable types of modules for urban search tasks has been presented. In this approach, an inventory of pre-existing hardware and software modules are assembled to quickly produce a system for natural and man-made disaster emergencies due to simplicity of modules. Depending on the mission, the operator would decide on the type of robot suitable for the mission. The analysis of forward and inverse kinematics from the presented $J$ module resulted in different types of locomotion; legged and wheeled robots may be assembled. The control motion modes attempt to facilitate the operator a better control for locomotion and manipulation. The operator may control the modular robot configuration in an easier and faster way. The modular robot system is designed, built and tested highlighting its module types, modeling and a few examples of locomotion tasks. Regarding different $S$ and $J$ modules types, the modular robot may locomote as a mobile or legged robot.

\section{ACKNOWLEDGMENTS}

The authors would like to thank CICYT and CONACYT for financing this research project. The SMART project has been financed within the Industrial Design and Production Program (DPI2003-00759 and DPI-2006-06493).

\section{REFERENCES}

[1] R. Snyder, "Robots assist in search and rescue efforts at WTC," IEEE Robot. Automat. Mag., vol. 8, pp. 26-28, 2001.

[2] R. Murphy, "Marsupial and shape-shifting robots for urban search and rescue," IEEE Intell. Syst., vol. 15, no. 3, pp. 14-19, 2000.
[3] R. R. Murphy and J. J. Martinez, "Lessons learned from the NSF REU site grant: multiple autonomous mobile robots for search and rescue applications," in Proc. IEEE Frontiers Education, vol. 3, pp.1378-1382. 1997.

[4] R. Jarvis, "An autonomous heavy duty outdoor robotic tracked vehicle," in Proceedings of the 1997 IEEE/RSJ International Conference on Intelligent Robots and Systems, vol. 1, 1997, pp. 1352-1359.

[5] S. Hirose, "Snake, walking and group robots for super mechanosystem," in Proc. IEEE SMC'99 Conf., vol. 2, pp.129-133, 1999.

[6] A. Kobayashi and K. Nakamura, "Rescue robot for fire hazards," in Proc. 1983 Int. Conf. Advanced Robotics, pp.91-98, 1983.

[7] H. Amano, K. Osuka, and T. Tarn, "Development of vertically moving robot with gripping handrails for fire fighting," in Proc. 2001 Int. Conf. Intelligent Robots and Systems, pp.661-662, 2001.

[8] D. McAndrew, A. Mangolds, and D. Deguire, "Tactical mobile robotic search and rescue mission modules," Proc. SPIE, vol. 4024, pp. 274 $282,2000$.

[9] A. Castano, W. M. Shen, and P. Will, "CONRO: Toward deployable robots with inter-robot metamorphic capabilities," Auton. Robots, vol.8, no. 3, pp.309-324, 2000.

[10] R. M.Voyles, "TerminatorBot:Arobot with dual-use arms for manipulation and locomotion," in Proc. Int. Conf. Robotics and Automation, pp. 61- 66,2000

[11] S. Tadokoro, R. Verhoeven, M. Hiller, and T. Takamori, "A portable parallel manipulator for search and rescue at large-scale urban earthquakes and an identification algorithm for the installation in unstructured environments," in Proc. Int. Conf. Intelligent Robots and Systems, pp.1222-1227, 1999.

[12] I. Eirkmen, A. M. Erkmen, F. Matsuno, R. Chatterjee, and T Kamegawa, "Snake robots to the rescue!," IEEE Robot. Automat. Mag., vol. 9, pp.17-25, 2002.

[13] J. S. Jennings, G. Whelan, and W. F. Evans, "Cooperative search and rescue with a team of mobile robots," in Proc.8th Int. Conf. on Advanced Robotics, pp.193-200, 1997.

[14] R. Masuda, T. Oinuma, and A. Muramatsu, "Multi-sensor control system for rescue robot," in Proc. Int. Conf. Multisensor Fusion and Integration for Intelligent Systems, pp.381-387, 1996.

[15] J. G. Blitch and R. Mauer, "KNOBSAR: A knowledge based system prototype for robot assisted urban search and rescue," Simulation, vol 66 , pp. $375-391,2000$.

[16] J. G. Blitch, "Artificial intelligence technologies for robot assisted urban search and rescue," Expert Syst. Applicat., vol. 11, no. 2, pp.109. $124,1996$.

[17] R. Murphy, J. Casper, M. Micire, and J. Hyams, "Mixed-initiative control of multiple heterogeneous robots for search and rescue," Tech. Rep., Univ. South Florida, Tampa, 2000

[18] M. Yim, D. Duff, K.D. Roufas, "Walk on the Wild Side," IEEE Robotics \& Automation Magazine, pp.4953, 2002.

[19] S. Murata, E. Yoshida, A. Kamimura, H. Kurokawa, K.Y. Tomita, S. Kokaji, "MTRAN: Self-Reconfigurable Modular Robotis System," IEEE/ASME Trans. Mechatronics, pp.431441, 2002.

[20] A. Castano, A. Behar, P.M. Will, "The CONRO Modules for Reconfigurable Robots," IEEE/ASME Trans. Mechatronics, pp.403409, 2002.

[21] W.-M. Shen, M. Krivokon, H. Chiu, J. Everist, M. Rubenstein, J. Venkatesh, "Multimode Locomotion for Reconfigurable Robots," Autonomous Robots, pp.165177, 2006.

[22] F. Aghili, K. Parsa, "A Reconfigurable Robot With Lockable Cylindrical Joints," IEEE Transactions on Robotics, vol.25, no.4, pp.785-797. 2009.

[23] S. Farritor, S. Dubowsky, N. Rutman, J. Cole, "A systems-level modular design approach to field robotics," in Proc. International Conference on Robotics and Automation, Vol. 4, pp.2890-2895, 1996.

[24] J. Casper, R.R. Murphy, "Human-robot interactions during the robotassisted urban search and rescue response at the World Trade Center," IEEE Transactions on Systems, Man, and Cybernetics, Part B: Cybernetics, vol.33, no.3, pp.367- 385, 2003.

[25] H.A. Yanco, J.L. Drury and J. Scholtz, "Beyond usability evaluation: analysis of human-robot interaction at a major robotics competition, Human-Computer Interaction, Vol. 19, No. 1 \& 2, pp.117 - 149, 2004 